\title{
Inhibition of pro-protein convertase subtilisin/kexin type 6 has a protective role against synovitis in a rat model of rheumatoid arthritis
}

\author{
HUIYU JIANG ${ }^{1-3}$, LIN WANG ${ }^{1-3}$ and JIHONG PAN ${ }^{1-3}$ \\ ${ }^{1}$ Shandong Medicinal Biotechnology Centre; ${ }^{2}$ Key Lab for Biotechnology Drugs of the Ministry of Health; \\ ${ }^{3}$ Key Lab of Rare and Uncommon Disease, Jinan, Shandong 250022, P.R. China
}

Received August 12, 2014; Accepted August 24, 2015

DOI: $10.3892 / \mathrm{mmr} .2015 .4376$

\begin{abstract}
The aim of the present study was to assess the effects of pro-protein convertase subtilisin/kexin type 6 (PCSK6), a proteinase implicated in the proteolytic activity of various precursor proteins and involved in the regulation of protein maturation, in fibroblast-like synoviocytes (FLS) of a rat model of collagen-induced arthritis (CIA). Cultured FLS from CIA models were subjected to small interfering RNA mediated PCSK6 knockdown, followed by assessment of the proliferation, invasive and migratory capacity, the secretion of inflammation factors and the cell cycle. Expression of genes associated with proliferation, invasion, migration and inflammation was detected by reverse transcription polymerase chain reaction. The results showed that PCSK6 knockdown significantly decreased the cell proliferation, invasion and migration of FLS from rats with CIA. ELISA showed an obvious decrease of tumor necrosis factor $\alpha$ and interleukin $1 \beta$ secretion, and flow cytometric analysis revealed G0/G1 arrest of FLS following PCSK6 knockdown. Furthermore, a decrease in the mRNA levels of inflammation-associated chemokine CXCL9, angiogenesis-associated genes MMP-2, MMP-9 and NOSTRIN, hypoxia-associated gene HIF-1 $\alpha$,
\end{abstract}

Correspondence to: Dr Jihong Pan, Shandong Medicinal Biotechnology Centre, 18877 Jingshi Road, Jinan, Shandong 250022, P.R. China

E-mail:pjh933@sohu.com

Abbreviations: PCSK6, pro-protein convertase subtilisin/kexin type 6; RA, rheumatoid arthritis; CIA, collagen-induced arthritis; FLS, fibroblast-like synoviocytes; IL, interleukin; TNF, tumor necrosis factor; siRNA, small interfering RNA; NOSTRIN, nitric oxide synthase trafficking; MMP9, matrix metallopeptidase 9; CXCL9, chemokine (C-X-C motif) ligand 9; HIF-1 $\alpha$, hypoxia inducible factor 1, $\alpha$ subunit; IGF-2, insulin-like growth factor 2; MPZL2, myelin protein zero-like 2; PADI4, peptidyl arginine deiminase, type IV

Key words: pro-protein convertase subtilisin/kexin type 6, rheumatoid arthritis, collagen-induced arthritis, fibroblast-like synoviocytes, small interfering RNA adhesion-associated gene MPZL2, proliferation-associated gene IGF-2 and citrullination-associated gene PADI4 was detected after PCSK6 knockdown. The results of the present study indicated that inhibition of PCSK6 may have a protective role against synovitis in rheumatoid arthritis.

\section{Introduction}

Type II collagen-induced arthritis (CIA) is a typical animal model of rheumatoid arthritis (RA), a systemic autoimmune disease characterized by chronic inflammation of the synovium and hyperplasia of synovial fibroblasts; this inflammation can erode adjacent cartilage and bone and cause subsequent joint destruction (1). This destructive process is at least partly mediated by fibroblast-like synoviocytes (FLS) from the synovium. Indeed, FLS from RA patients were shown to attach to and invade normal cartilage in a SCID mouse co-implementation model (2). Furthermore, FLS are implicated in all aspects of the pathogenesis of RA (3). Thus, FLS in the rheumatoid synovium are known to be aggressive and highly proliferative, and may attack the cartilage, possessing characteristics similar to those of transformed cells (3), including anchorage-independent growth (4), insensitivity to apoptosis and enhanced proliferation, and are able to invade the cartilage (5). The CIA model exhibits numerous clinical similarities with human RA (6).

Cultured FLS express high levels of proteinases, which are able to degrade extracellular matrix components, including collagens. One family of proteinases expressed by FLS are the matrix metalloproteinases (MMPs). FLS express MMP-1, -2, $-3,-9$ and -10 , and the expression levels of these MMPs are correlated with their invasiveness (7). However, MMPs are inactive precursors that must be processed by pro-protein convertases (PCs), which cleave single basic or paired basic residues of the pro-proteins to produce biologically active proteins. To date, nine PCs have been identified: PC1/PC3, PC2, pro-protein convertase subtilisin/kexin type 6 (PCSK6)/PACE4, PC4, PC5/PC6, PC7/PC8/LPC, furin, PCSK8 and PCSK9.

Furin is highly expressed in the synovium of RA patients and mice with CIA, and may protect against RA (8). PCSK6, however, has a major role in promoting the progression of prostate tumors to a status of increased aggressiveness (9). Of note, tumor tissues and the synovium of rats with CIA or 
patients with RA share common features, including excessive angiogenesis and fibrin deposition, de-regulation of cell proliferation and high coagulation activity (10). Thus, PCSK6 was suggested to have an important role in CIA, which differs from that of furin. Furthermore, a variant of PCSK6 was highly associated with reduced pain in knee osteoarthritis, providing a possible explanation as to why in the presence of an identical structural damage, certain individuals developed chronic pain, while others were protected. Studies on PCSK6-null mice also implicated PCSK6 in pain (11). However, a role for this protein in CIA or RA has not yet been reported.

The present study investigation of the role of PCSK6 in synovitis of rats with CIA. For this, FLS were isolated from the synovium of a rat model of CIA, and PCSK6 knockdown was performed in isolated FLS to identify changes in their proliferation, migratory and invasive capacity, and cell cycle progression.

\section{Materials and methods}

Induction of CIA. Ten male Wistar rats (age, 6-8 weeks; weight 100-120 g) susceptible to developing CIA were purchased from Vital River Laboratories (Beijing, China). All rats were kept under controlled environmental conditions with a mean temperature of $22 \pm 3^{\circ} \mathrm{C}, 12 \mathrm{~h}$ dark-light cycle, relative humidity of $40 \%$ and access to food and water ad libitum. The experimental protocol was approved by the Ethics Committee for the use of animals of Shandong Academy of Medical Science (Jinan, China). All efforts were made to minimize discomfort and reduce the number of experimental animals used. All procedures conformed to the ethical guidelines regarding the care and use of laboratory animals, published by the International Association for the Study of Pain and the National Institutes of Health (12). After acclimatization for one week in their cages, the rats received a sub-cutaneous injection of $100 \mu \mathrm{g}$ native bovine type II collagen (Chondrex, Inc., Redmond, WA, USA) emulsified in Freund's complete adjuvant (Chondrex, Inc.) into the base of the tail. A second sub-cutaneous boost of $100 \mu \mathrm{g}$ type II collagen in Freund's complete adjuvant was given 21 days later. The animals were anesthetized followed by a lethal dose of sodium pentobarbital (Euthasol; Virbac USA, Fort Worth, TX, USA) 20 days after the second immunization when the paws were notably swollen.

Culture and identification of synovial fibroblasts. The synovial tissues of rats with CIA was finely chopped and incubated with type II collagenase $(1 \mathrm{mg} / \mathrm{ml}$; Sigma-Aldrich, St. Louis, MO, USA) in Dulbecco's modified Eagle's medium (DMEM, HyClone, Thermo Fisher Scientific, Waltham, MA, USA) for $6 \mathrm{~h}$ in an incubator at $37^{\circ} \mathrm{C}$ with $5 \% \mathrm{CO}_{2}$. The tissue was treated with $0.25 \%$ trypsin (Solabio, Beijing, China) in phosphate-buffered saline (PBS), in a volume equivalent to that of DMEM. Cells were filtered and cultured overnight in DMEM supplemented with $10 \%$ fetal bovine serum (FBS; HyClone) as well as penicillin (100 IU/ml) and streptomycin (100 $\mu \mathrm{g} / \mathrm{ml})$ (Gibco-BRL, Invitrogen Life Technologies, Carlsbad, CA, USA) for three passages. FLS of rats with CIA at passage 4-6 were used for the present study, which were negative for CD14, CD3, CD19 and CD56 expression as identified by flow cytometric analysis using a Coulter Epics XL flow cytometer (Beckman Coulter, Brea, CA, USA). Phycoerythrin (PE)-conjugated CD14 (cat. no. 12-0149), fluorescein isothiocyanate (FITC)-conjugated CD3 (cat. no. 11-0039), FITC-conjugated CD19 (cat. no. 11-0199) and PE-conjugated CD56 (cat. no. 12-0567) antibodies were obtained from eBioscience Inc. (San Diego, CA, USA) and used at a 1:50 dilution.

Inhibition of PCSK6 expression with small interfering (si)RNAs. siRNA targeting PCSK6 (target mRNA sequence: 5'-GCAGAG AAGAAUGUAUUCATT-3') was designed and synthesized by GenePharma Co. Ltd (Shanghai, China). Cultured FLS were transfected with siRNA at $160 \mathrm{nmol} / \mathrm{l}$ using a HiPerFect transfection reagent (Qiagen, Hilden, Germany) according to the manufacturer's instructions. The cells were harvested for analysis at $24 \mathrm{~h}$ following the transfection. A negative siRNA (sequence: 5'-UUCUCCGAACGUGUCACGUTT-3') was designed and synthesized by Shanghai GenePharma Co., Ltd. (Shanghai, China). was used as the negative control; treatment with transfection reagent only was used as the Mock group.

Reverse transcription-quantitative polymerase chain reaction $(R T-q P C R)$. Total RNA was extracted from the cultured cells and the human tissue using a Total RNA kit (R6834; Omega Bio-Tek, Inc., Norcross, GA, USA) and reverse-transcribed using a ReverTra Ace qPCR RT kit (FSQ-101; Toyobo, Osaka, Japan) according to the manufacturer's instructions. qPCR was performed using the LightCycler 480 (4887352001; Roche Diagnostics, Basel, Switzerland) using the following amplification protocol: Denaturation at $95^{\circ} \mathrm{C}$ for $10 \mathrm{~min}$, followed by 40 cycles of denaturation at $95^{\circ} \mathrm{C}$ for $10 \mathrm{sec}$ followed by annealing at $60^{\circ} \mathrm{C}$ for $1 \mathrm{~min}$ and extension at $72^{\circ} \mathrm{C}$ for $1 \mathrm{sec}$. The comparative threshold cycle $(\mathrm{Ct})$ method was used to analyze the relative expression of mRNA (13). The relative target gene expression was normalized to GAPDH mRNA levels. Primers for qPCR were also designed according to the consensus sequence as determined using AlignX (Vector NTI Advance 11.0; Invitrogen Life Technologies). The primers for the amplification of PCSK6 were as follows: Forward, 5'-ACTCCAGAAGAAGAGGAAG AGTA-3' and reverse, 5'-ACCATCGCAGCCTTTATCA-3'. The primers for the amplification of GAPDH were as follows: Forward, 5'-TGAACGGGAAGCTCACT-3' and reverse, 5'-CAT GTCAGATCCACAACGGATA-3'. All primers are synthesized by Bio-Asia Diagnostics Co., Ltd. (Shanghai, China). For all PCRs, standard curves, dissociation curves and migration of PCR products on acrylamide gels were performed to confirm the specificity of the products. The specificity of the qPCR assay was evaluated by melting curve analysis, which showed that the PCSK6 amplification product generated a melting peak at $81.20 \pm 0.34^{\circ} \mathrm{C}$ without primer-dimers or non-specific products.

Cell proliferation assay. The CIA FLS were seeded onto 96-well culture plates and cultured at $37^{\circ} \mathrm{C}$ to $80 \%$ confluence. The cultures were treated with PCSK6 siRNA (160 nmol/l). After incubation for 24,48 or $72 \mathrm{~h}, 20 \mu 15 \mathrm{mg} / \mathrm{ml}$ MTT in PBS was added to each well, and cultures were incubated for $4 \mathrm{~h}$ at $37^{\circ} \mathrm{C}$ in the incubator. MTT solution was removed and $150 \mu \mathrm{l}$ dimethyl sulfoxide was added to extract the MTT-formazan products at room temperature for $10 \mathrm{~min}$. The absorbance was measured in triplicate at $490 \mathrm{~nm}$ using a spectrophotometer (DNM-9602G; Prolong Group, Beijing, China). 
Cell invasion assay and migration assays. The invasive ability of the cells was tested using Transwell plates (BD Biosciences, Franklin Lakes, NJ, USA). FLS were seeded into the upper chamber of the Transwell plate at a density of $3 \times 10^{4}$ cells/well and incubated at $37^{\circ} \mathrm{C}$ with $160 \mathrm{nmol} / \mathrm{l}$ siRNA for $8 \mathrm{~h}$. The upper and the lower chambers were then filled with medium without FBS, followed by incubation for $12 \mathrm{~h}$. Subsequently, the lower chamber was filled with $20 \%$ FBS in DMEM, followed by a further incubation for $24 \mathrm{~h}$. The non-invaded cells at the upper surface of the membrane were removed with cotton swabs and the invaded cells on the lower side were stained with Giemsa (Solabio). The cell number of cells that had transgressed through the filter was quantified in five random fields at $\mathrm{x} 100$ magnification and the average number was calculated. Cells were observed using a XDS-1B microscope (Nikon, Tokyo, Japan)

A wound-healing assay was performed to test the migration ability of FLS. Cells were plated onto 24-well plates and cultured at $37^{\circ} \mathrm{C}$ until reaching $80 \%$ confluence. The cell monolayers were scratched linearly in multiple areas with a cell scraper (Corning Life Sciences, Tewksbury, MA, USA), and cells were subsequently transfected with $160 \mathrm{nmol} / 1$ PCSK6 siRNA or control siRNA. After $24 \mathrm{~h}$ of incubation, the number of cells migrated into the scratched area was calculated using a method identical to that described above.

Determination of interleukin (IL)-6,IL-1 $\alpha, I L-1 \beta, I L-17$ and tumor necrosis factor (TNF)- $\alpha$ levels by ELISA. FLS were transfected with $160 \mathrm{nmol} / 1 \mathrm{siRNA}$ for $24 \mathrm{~h}$, and the culture medium was collected and centrifuged at $800 \mathrm{x}$ g for $5 \mathrm{~min}$ at $4^{\circ} \mathrm{C}$. $100 \mu \mathrm{l}$ medium was added to a 96-well microplate (Corning-Costar, Corning, NY, USA), which was stored overnight at $4^{\circ} \mathrm{C}$. After gently washing with PBS containing Tween 20 (Solabio, Beijing, China), 1\% bovine serum albumin (Solabio) plus 5\% sucrose (Solabio) was used for blocking for $1 \mathrm{~h}$ at $37^{\circ} \mathrm{C}$. Following three washes with PBS, antibodies against IL- $1 \alpha$, IL-1 $\beta$, IL-17 and TNF- $\alpha$ (all from Abcam, Cambridge, MA, USA; dilution, 1:1,000) were applied to the plate for overnight incubation. The plate was washed, blocked and incubated with a 1:2,000 dilution of horseradish peroxidase-conjugated anti-rabbit immunoglobulin $\mathrm{G}$ (ProteinTech, Chicago, IL, USA) for $3 \mathrm{~h}$ at $37^{\circ} \mathrm{C}$. Staining was developed using a TMB kit (CW0050; CWBIO, China). The absorbance at $450 \mathrm{~nm}$ was measured using a plate reader (Synergy HT; BioTek, Winooski, VT, USA). FLS treated with transfection reagent only were used as a control.

Cell cycle analysis. Cells were seeded onto six-well culture plates (Corning-Costar) at $1.0 \times 10^{6}$ cells/well and the cells were treated with siRNA as described above. After removal of the culture medium, the cells were harvested at $24 \mathrm{~h}$ by trypsinization, washed twice with ice-cold PBS and fixed overnight with $70 \%$ ethanol at $4^{\circ} \mathrm{C}$. Prior to analysis, the fixed cells were rinsed with PBS, re-suspended in PBS and stained for $30 \mathrm{~min}$ at $37^{\circ} \mathrm{C}$ with $1 \mathrm{ml} 0.05 \mathrm{mg} / \mathrm{ml}$ propidium iodide solution (Digguo Biotech, Beijing, China) containing $10 \mu \mathrm{g} / \mathrm{ml}$ RNase (Sigma-Aldrich) for $30 \mathrm{~min}$ at $37^{\circ} \mathrm{C}$. Cells were analyzed using a Coulter Epics XL flow cytometer (Beckman Coulter, Brea, CA, USA) and the DNA content was determined using EXPO32 software (Beckman Coulter).
Statistical analysis. All results were confirmed in at least three independent experiments and were expressed as the mean \pm standard deviation. Multiple comparisons were performed using one-way analysis of variance. All statistical analyses were performed using SPSS 17.0 software (SPSS, Inc., Chicago, IL, USA). $\mathrm{P}<0.05$ was considered to indicate a statistically significant difference between values.

\section{Results}

Knockdown of PCSK6 affects the proliferation, migration, and invasion of FLS from rats with CIA in vitro. Since the present study hypothesized that PCSK6 may be involved in the progression of RA, RNA interference was used to knockdown PCSK6 expression in order to assess the resulting effects on FLS from rats with CIA. PCSK6 expression was determined by RT-qPCR $24 \mathrm{~h}$ after transfection with either control siRNA or PCSK6 siRNA. The mRNA expression of PCSK6 was significantly decreased following transfection with PCSK6 siRNA $(\mathrm{P}=0.0001)$ (Fig. 1A), demonstrating effective knockdown. To evaluate the roles of PCSK6 in the proliferation, migration and invasion of FLS in RA, MTT, wound healing and Transwell assays were performed after PCSK6 knockdown. Transfection with siRNA-PCSK6 reduced the proliferation of FLS from rats with CIA compared to that of the control- or Mock-transfected cells with normal PCSK6 expression ( $\mathrm{P}=0.0001)$ (Fig. 1B). In the cell migration assay, monolayers of FLS were scratched and subsequently incubated with siRNA-PCSK6, control siRNA or transfection reagent only for $24 \mathrm{~h}$. Significantly fewer FLS were present in the wounded area when PCSK6 was knocked down $(\mathrm{P}=0.0001)$ (Fig. 1C). Furthermore, the invasion assay showed that significantly fewer FLS transgressed through the Transwell filter when PCSK6 was knocked down ( $\mathrm{P}=0.0001)$ (Fig. 1D).

Knockdown of PCSK6 decreases TNF- $\alpha$ secretion in FLS from rats with CIA. As RA is a chronic inflammatory condition, pro-inflammatory cytokines have prominent roles in the disease. In particular, TNF- $\alpha$, IL-1 $\beta$ and IL-17 are important pro-inflammatory cytokines associated with synovitis and joint destruction (5). TNF- $\alpha$, IL-1 $\alpha$, IL-1 $\beta$ and IL-17 levels were compared in the supernatant of FLS following treatment with $160 \mathrm{nmol} / \mathrm{l}$ PCSK6 siRNA or control siRNA. The secretion of IL- 6 , TNF- $\alpha$ and IL-1 $\beta$ by siRNA-PCSK6-transfected FLS was significantly lower as compared with that in the controls $(\mathrm{P}=0.0001)$ (Fig. 1E).

Cell cycle arrest in FLS from rats with CIA following PCSK6 knockdown. To further study the effects of PCSK6 on the proliferation of FLS from rats with CIA, flow cytometric cell cycle analysis was performed. The ratio of $\mathrm{G}_{0} / \mathrm{G}_{1}$-phase cells was significantly higher in FLS with PCSK6 knockdown as compared with that in the controls after 12,48 and $72 \mathrm{~h}$ (Fig. 2A), which suggested that downregulation of PCSK6 inhibited the cell cycle in FLS of mice with CIA.

Knockdown of PCSK6 induces downregulation of genes associated with proliferation, invasion, migration and inflammation. To gain further insight into the role of PCSK6 in the pathology of RA, the expression levels of genes associated with proliferation, invasion, migration and inflammation were detected 
A

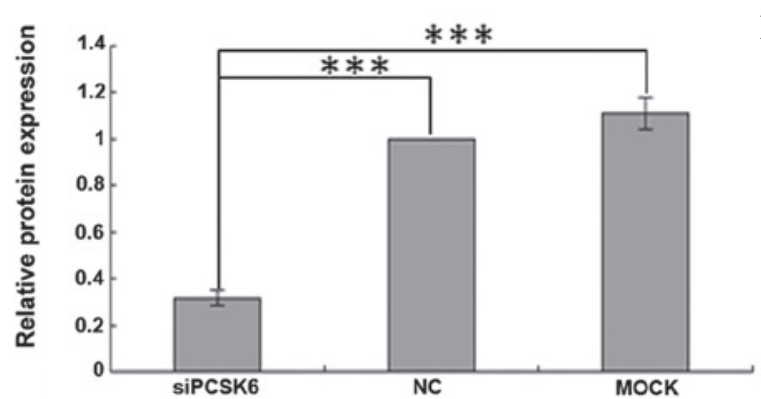

B

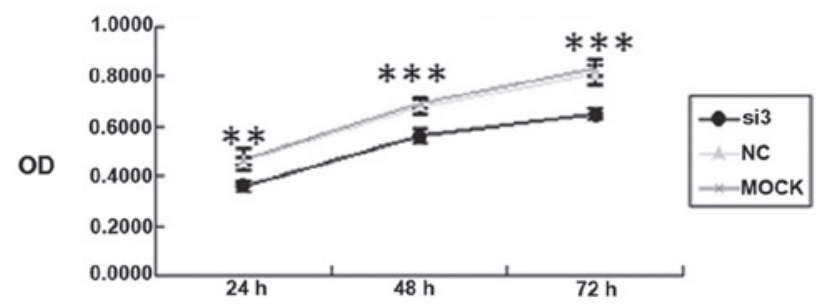

C
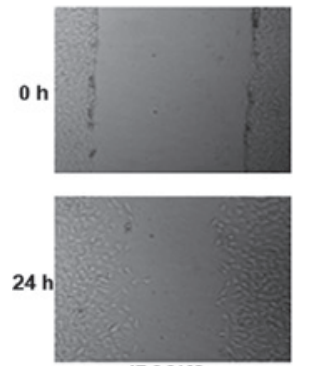

siPCSK6
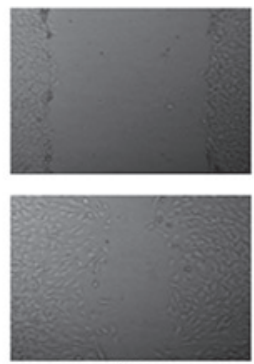

NC
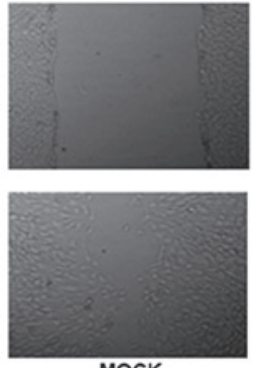

MOCK

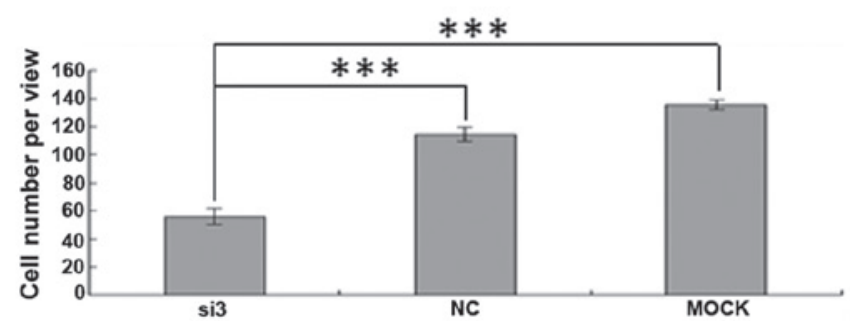

D
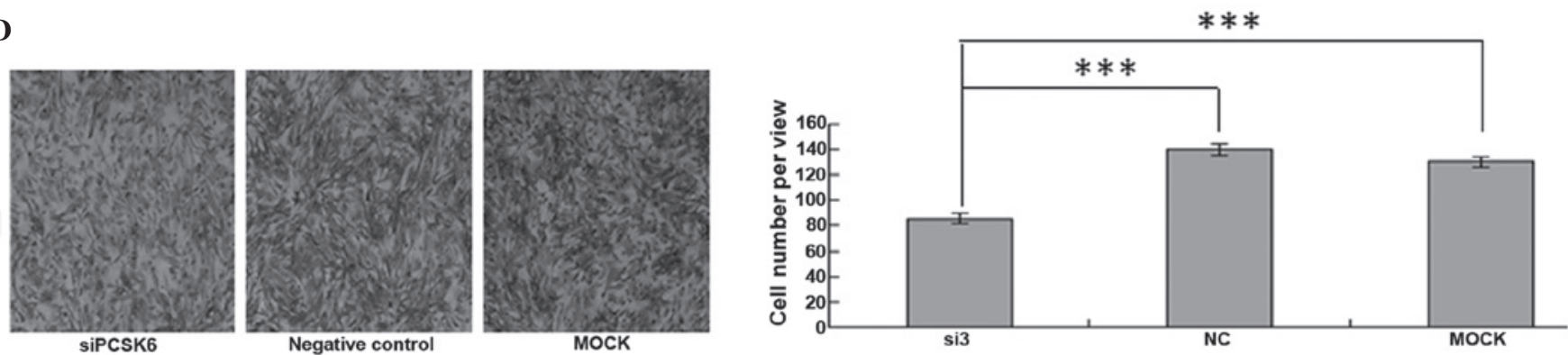

$\mathbf{E}$

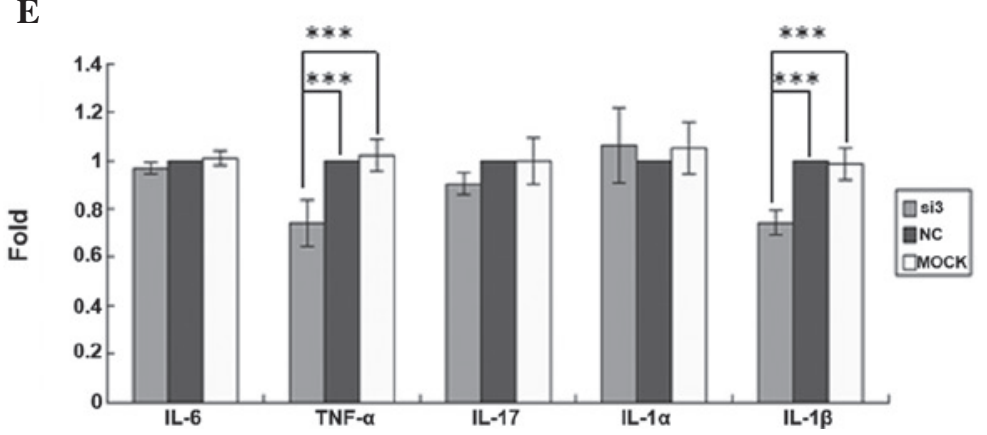

Figure 1. Synovial fibroblasts of a rat model of collagen-induced arthritis were transiently transfected with siRNA targeting PCSK6. (A) Knockdown of PCSK6 was confirmed by reverse-transcription polymerase chain reaction. (B) Cell proliferation was assessed using an MTT assay. (C) A wound healing assay was used to assess cell migration (magnification, x100). (D) The invasive ability was assessed using a Transwell assay and the average number of cells invaded through the filter following $24 \mathrm{~h}$ of incubation was quantified (magnification, x100). (E) Cytokine levels of IL-6, IL-1 $\alpha$, IL-1 $\beta$, IL-17 and TNF- $\alpha$ were assessed using ELISA. Values are expressed as the mean \pm standard deviation. Three independent experiments were performed for all of the above assays. ${ }^{* * *} \mathrm{P}<0.05$. NC, negative control; MOCK, control treated with transfection reagent only; si3, group transfected with anti-PCSK6 siRNA. Three independent experiments were conducted for each assay. IL, interleukin; siRNA, small interfering RNA; PCSK6, pro-protein convertase subtilisin/kexin type 6; TNF, tumor necrosis factor; NC, negative control; OD, optical density.

by RT-qPCR. A total of eight genes were found to be downregulated after PCSK6 knockdown (Fig. 3). Among these genes, IGF-2 was associated with cell proliferation, while CXCL9 was associated with inflammation. Other downregulated genes including, NOSTRIN, MMP2 and MMP9, were associated with angiogenesis, while MPZL2 is involved in cell adhesion. Of note, HIF-1 $\alpha$ and PADI4, which are closely associated with hypoxia, the main contributor to RA and CIA $(14,15)$, were also downregulated after PCSK6 knockdown.
The present study also examined the expression of Furin, which has a protective role in immune response-induced arthritis (8), following PCSK6 knockdown. However, silencing of PCSK6 had no significant effect on the expression of Furin.

\section{Discussion}

PCSK6 is one of the neuroendocrine-specific mammalian subtilisin-associated endoproteases (16), which contains a 

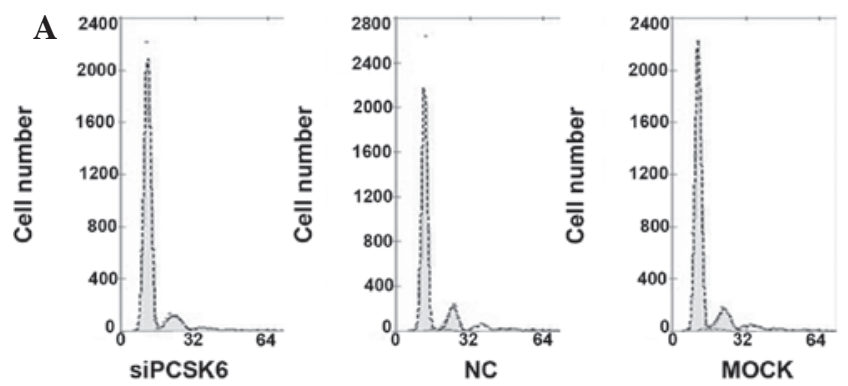

$\mathbf{B}$

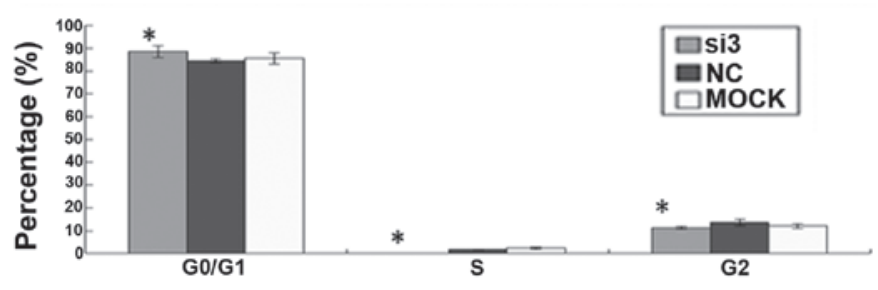

Figure 2. Effect of anti-PCSK6 siRNA on the cell cycle of synovial fibroblasts of a rat model of collagen-induced arthritis. (A) Representative flow cytometric cell cycle distribution graphs. (B) Statistical analysis of cell cycle phase populations. Values are expressed as the mean \pm standard deviation of three independent experiments. "P<0.05 vs. NC group. NC, negative control; MOCK, control treated with transfection reagent only; si3, group transfected with anti-PCSK6 siRNA. siRNA, small interfering RNA; PCSK6, pro-protein convertase subtilisin/kexin type 6.

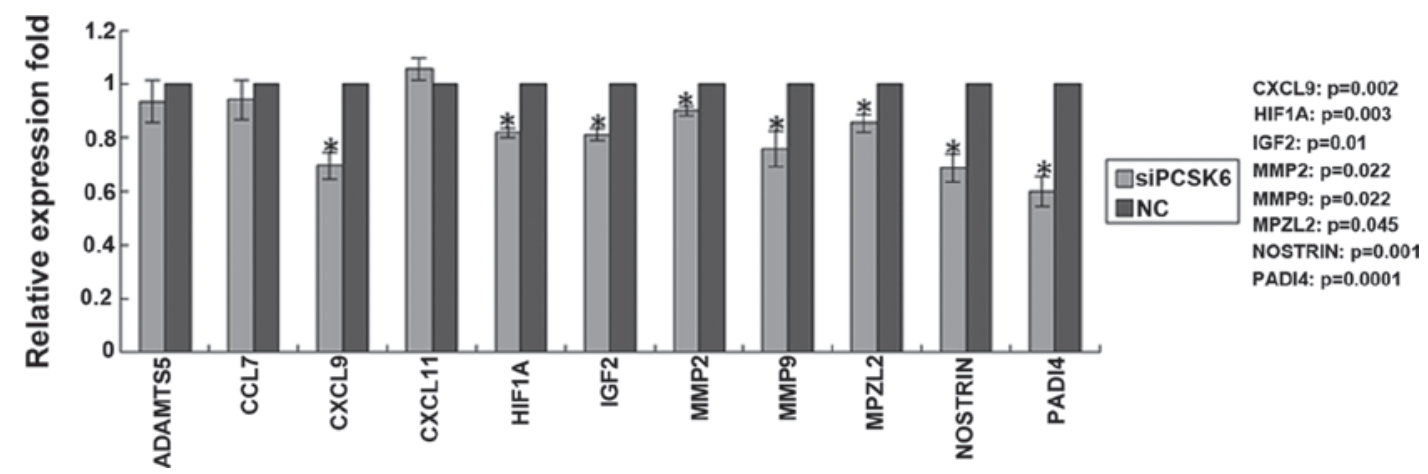

Figure 3. Gene expression in synovial fibroblasts of a rat model of collagen-induced arthritis was detected by reverse-transcription polymerase chain reaction GAPDH was used as an internal control. Values are expressed as the mean \pm standard deviation. siPCSK6, group transfected with small interfering RNA against pro-protein convertase subtilisin/kexin type 6; NC, negative control.

C-terminal cysteine-rich region (17), and which is thought to function in the secretory pathway.

Hyperplasia of synovial fibroblasts contributes to the pathogenesis of RA and is capable of eroding adjacent cartilage and bone and causing subsequent joint destruction. As PCSK6 had been indicated to have a critical role in tumor progression (7), cell proliferation, migration and invasion, the present study attempted to determine the function of PCSK6 in FLS of rats with CIA. Of note, significant decreases in the proliferation as well as in the migratory and invasive capacities were observed in FLS from rats with CIA after PCSK6 silencing. This suggested that PCSK6 has an important role in the hyperplasia and erosion capacity of FLS.

CIA is a typical model of RA, which is characterized as a chronic inflammatory disease. IL-6, TNF- $\alpha$, IL-1 $\beta$ and IL-17 are important pro-inflammatory cytokines in CIA, which are associated with synovitis and joint destruction. In the present study, knockdown of endogenous PCSK6 led to reduced TNF $\alpha$ and IL- $1 \beta$ expression in FLS from rats with. TNF- $\alpha$ is known to promote fibrosis of synovial tissues as well as immune responses (18). It has an important role in the growth and differentiation of numerous types of normal cell and forms a complex immune network with other cytokines during the inflammatory process (19). Joosten et al (20) reported that in murine collagen-induced arthritis, inflammation and cartilage degradation were inhibited by blocking IL-1 $\beta$. The present study found that PCSK6 knockdown led to decreases in IL-1 $\beta$ and TNF- $\alpha$ levels in FLS of rats with CIA, which suggested that PCSK6 exacerbates inflammation by regulating the expression or secretion of cytokines, including IL-1 $\beta$ and TNF- $\alpha$, in FLS during RA.

To elucidate the roles of PCSK6 in FLS during RA, the present study analyzed the expression levels of several genes which are known to be associated with inflammation, proliferation, invasion, migration and hypoxia after knockdown of PCSK6 in FLS from rats with CIA. Among them, CXCL9 is a sensitive marker for disease activity in patients with RA (21). In the present study, downregulation of CXCL9 expression was observed in FLS of rats with CIA following knockdown of PCSK6. This result confirmed that PCSK6 regulates the proliferation and migration of FLS through chemokines such as CXCL9.

MMPs are secreted or membrane-anchored zinc-dependent endopeptidases, which have been shown participate in the initiation of cell movement in the extracellular matrix and to be able to degrade most extracellular matrix proteins, particularly type IV collagen, the major component of basement membranes (7). Re-modeling of the extracellular matrix by MMPs is important in angiogenesis. As abnormal angiogenesis in the synovium is a key characteristic of CIA, factors which stimulate MMP expression may have a role in CIA. In the present study, a marked decrease of MMP-2 and MMP-9 expression was observed in FLS of mice with CIA after PCSK6 knockdown, which is consistent to the function of PCSK6 in processing pre-mature MMP-2/9 to mature types a reported by Mahloogi et al (15). MMPs participate in angiogenesis by degrading and remodelling the extracellular matrix and basement membranes, allowing activated endothelial cells to proliferate and migrate, as well as 
releasing extracellular matrix-bound growth factors, including fibroblast growth factor-2, vascular endothelial growth factor or insulin-like growth factor-1 (22). NOSTRIN is another type of protein with an important role in developmental angiogenesis (23). In the present study, NOSTRIN mRNA was detected to be downregulated after PCSK6 knockdown in FLS of mice with CIA. This suggested that PCSK6 may have an important role in angiogenesis during CIA through its impact on NOSTRIN and MMP-9.

As mentioned above, hypoxia of synovial tissues in inflamed joints is one of the most important characteristics of RA/CIA. Hypoxia-inducible factor $1 \alpha(\mathrm{HIF}-1 \alpha)$, the key transcriptional factor in the hypoxic response, is upregulated in RA (24). RA is thought to decrease the oxygen supply, leading to synovial hypoxia and hypo-perfusion (25). The present study we found that HIF-1 $\alpha$ expression was regulated, either directly or indirectly, by PCSK6. Therefore, it was hypothesized that PCSK6 may be involved in the regulation of the hypoxic synovial microenvironment.

Another important member of the pro-protein convertase family, furin, has been previously reported to have protective roles in immune response-induced arthritis (8); therefore, the present study also tested the expression of furin after PCSK6 knockdown. However, the results showed that knockdown of PCSK6 did not affect the expression of furin. According to the in vitro results of the present study, the role of PCSK6 in CIA is opposite to that reported for furin.

In conclusion, the present study demonstrated that PCSK6 may have important roles in the proliferation, migration, invasion and angiogenesis of FLS during RA/CIA. In addition, PCSK6 contributes to the secretion of pro-inflammatory cytokines, hypoxia of FLS and deregulation of the cell cycle of FLS in RA/CIA. The results of the present study indicated that inhibition of PCSK6 may have a protective role against synovitis in RA.

\section{Acknowledgements}

The present study was supported by the National Natural Science Foundation of China (NSFC) (no. 81102275), the Natural Science Foundation of Shandong Province (no. ZR2011CQ028), the National Basic Research Program of China (no. 2010CB529105) and the Shandong Science and Technology Research Program (no. 2012GSF12115).

\section{References}

1. Sokka T: Work disability in early rheumatoid arthritis. Clin Exp Rheumatol 21 (5 Suppl 31): S71-S74, 2003.

2. Muller-Ladner U, Kriegsmann J, Franklin BN, Matsumoto S, Geiler T, Gay RE and Gay S: Synovial fibroblasts of patients with rheumatoid arthritis attach to and invade normal human cartilage when engrafted into SCID mice. Am J Pathol 149: 1607-1615, 1996

3. Tolboom TC, van der Helm-van Mil AH, Nelissen RG, Breedveld FC, Toes RE and Huizinga TW: Invasiveness of fibroblast-like synoviocytes is an individual patient characteristic associated with the rate of joint destruction in patients with rheumatoid arthritis. Arthritis Rheum 52: 1999-2002, 2005.

4. Lafyatis R, Remmers EF, Roberts AB, Yocum DE, Sporn MB and Wilder RL: Anchorage-independent growth of synoviocytes from arthritic and normal joints: Stimulation by exogenous platelet-derived growth factor and inhibition by transforming growth factor-and retinoids. J Clin Invest 83: 1267-1276, 1989.
5. Bartok B and Firestein GS: Fibroblast-like synoviocytes: Key effector cells in rheumatoid arthritis. Immunol Rev 233: 233-255, 2010.

6. Holmdahl R, Mo J, Nordling C, Larsson P, Jansson L, Goldschmidt T, Andersson M and Klareskog L: Collagen induced arthritis: An experimental model for rheumatoid arthritis with involvement of both DTH and immune complex mediated mechanisms. Clin Exp Rheumatol 7 (Suppl 3): S51-S55, 1989.

7. Tolboom TC, Pieterman E, van der Laan WH, Toes RE, Huidekoper AL, Nelissen RG, Breedveld FC and Huizinga TW: Invasive properties of fibroblast-like synoviocytes: Correlation with growth characteristics and expression of MMP-1, MMP-3, and MMP-10. Ann Rheum Dis 61: 975-980, 2002.

8. Lin H, Ah Kioon MD, Lalou C, Larghero J, Launay JM, Khatib AM and Cohen-Solal M: Protective role of systemic furin in immune response-induced arthritis. Arthritis Rheum 64: 2878-2886, 2012.

9. D'Anjou F, Routhier S, Perreault JP, Latil A, Bonnel D, Fournier I, Salzet M and Day R: Molecular validation of PACE4 as a target in prostate cancer. Transl Oncol 4: 157-172, 2011.

10. Chang $X$ and Han J: Expression of peptidylarginine deiminase type 4 (PAD4) in various tumors. Mol Carcinog 45: 183-196, 2006.

11. Malfait AM, Seymour AB, Gao F, Tortorella MD, Le Graverand-Gastineau MP, Wood LS, Doherty M, Doherty S, Zhang W and Arden NK: A role for PACE4 in osteoarthritis pain: Evidence from human genetic association and null mutant phenotype. Ann Rheum Dis 71: 1042-1048, 2012.

12. National Research Council: Guide for the Care and Use of Laboratory Animals. 8th edition, National Academies Press, Washington, DC, 2011.

13. Livak KJ and Schmittgen TD: Analysis of relative gene expression data using real-time quantitative PCR and the 2(-Delta Delta c(T)) method. Methods 25: 402-408, 2001.

14. Choi HM, Lee YA, Lee SH, Hong SJ, Hahm DH, Cho SY, Yang HI, Yoo MC and Kim KS: Adiponectin may contribute to synovitis and joint destruction in rheumatoid arthritis by stimulating vascular endothelial growth factor, matrix metalloproteinase-1, and matrix metalloproteinase-13 expression in fibroblast-like synoviocytes more than proinflammatory mediators. Arthritis Res Ther 11: R161, 2009.

15. Mahloogi H, Bassi DE and Klein-Szanto AJ: Malignant conversion of non-tumorigenic murine skin keratinocytes overexpressing PACE4. Carcinogenesis 23: 565-572, 2002.

16. Mains RE, Berard CA, Denault JB, Zhou A, Johnson RC and Leduc R: PACE4: A subtilisin-like endoprotease with unique properties. Biochem J 321: 587-593, 1997.

17. Seidah NG, Chrétien M and Day R: The family of subtilisin/kexin like pro-protein and pro-hormone convertases: Divergent or shared functions. Biochimie 76: 197-209, 1994.

18. Lee A, Qiao Y, Grigoriev G, Chen J, Park-Min KH, Park SH, Ivashkiv LB and Kalliolias GD: Tumor necrosis factor alpha induces sustained signaling and a prolonged and unremitting inflammatory response in rheumatoid arthritis synovial fibroblasts. Arthritis Rheum 65: 928-938, 2013.

19. Feldmann M and Maini RN: Lasker clinical medical research award: TNF defined as a therapeutic target for rheumatoid arthritis and other autoimmune diseases. Nat Med 9: 1245-1250, 2003.

20. Joosten LA, Helsen MM, Van deLoo FA and Van deBerg WB: Anticytokine treatment of established type II collagen-induced arthritis in DBA/1 mice. A comparative study using anti-TNF alpha, anti-IL-1 alpha/beta and IL-1Ra. Arthritis Rheum 39: 797-809, 1996.

21. Kuan WP, Tam LS, Wong CK, Ko FW, Li T, Zhu T and Li EK: CXCL 9 and CXCL 10 as sensitive markers of disease activity in patients with rheumatoid arthritis. J Rheumatol 37: 257-264, 2010.

22. Kovacevic I, Hu J, Siehoff-Icking A, Opitz N, Griffin A, Perkins AC, Munn AL, Müller-Esterl W, Popp R, Fleming I, et al: The F-BAR protein NOSTRIN participates in FGF signal transduction and vascular development. EMBO J 31: 3309-3322, 2012.

23. Pardo A and Selman M: Matrix metalloproteases in aberrant fibrotic tissue remodeling. Proc Am Thorac Soc 3: 383-388, 2006.

24. Hollander AP, Corke KP, Freemont AJ and Lewis CE: Expression of hypoxia-inducible factor 1alpha by macrophages in the rheumatoid synovium: Implications for targeting of therapeutic genes to the inflamed joint. Arthritis Rheum 44: 1540-1544, 2001.

25. Muz B, Khan MN, Kiriakidis S and Paleolog EM: Hypoxia. The role of hypoxia and HIF-dependent signaling events in rheumatoid arthritis. Arthritis Res Ther 11: 201, 2009. 